\title{
55 Year Old Woman With Pneumonia
}

\author{
Andra Popescu, $M D$
}

A 55 year old Caucasian female smoker, with past medical history significant for hypertension and peptic ulcer disease presented to the emergency department with a four day complaint of malaise, fever, and myalgias. She was seen by her primary care physician who started her on amoxicillin clavulanate for possible strep throat infection. Her family reported a productive cough with green sputum, pleuritic chest pain, nausea, vomiting and diarrhea for one week. They also felt she was getting more confused over the past several hours.

The patient was found to be confused, hypotensive, and hypoxic. Temp $98.1 \mathrm{~F}$, heart rate 107 beats per minute, respiratory rate 16 breaths per minute, blood pressure $73 / 51 \mathrm{mmHg}$, and oxygenating at $89 \%$ on room air. Physical exam revealed bilateral wheezing that was more prominent in the right lung field. She was tachycardic with no murmurs or rubs. The abdomen was soft and non-tender with no organomegaly and she had no evidence of a rash.

Labs on admission: WBC 2,600/mm3, Hemoglobin $13.3 \mathrm{~g} / \mathrm{dL}$, platelets 255,000/mm3, Na+134, K+ 4.5, HCO3-19, BUN/Cr 49/2.8, AST 85, ALT 53. ABG: pH 7.2, PaCO2 49, PaO2 75, O2 Sat $92 \%$

The differential diagnoses at the time of presentation included community acquired pneumonia with Streptococcus pneumoniae, Haemophilus influenzae, and Moraxella catharalis being high on the list of pathogens along with the atypical organisms such as Legionella, Mycoplasma and Chlamydia. Other infectious pathogens such as anaerobes and fungi are also included. Non-infectious etiologies including pulmonary embolism, vasculitis, hypersensitivity pneumonitis and neoplastic lymphangitis spread were also considered.

The patient was admitted to the ICU with presumptive admission diagnosis of septic shock secondary to pneumonia and was intubated within 1 hour of admission due to hypoxic respiratory failure requiring FIO2 100\% and 16+ PEEP to maintain PO2 of $70 \mathrm{mmHg}$. Stress dose steroids were started empirically, as well as empiric antibiotic coverage with azythromicin, pipericillin/tazobactam, and vancomycin. Recombinant human activated protein $\mathrm{C}$ was also administered 12 hours after admission. Two days later urine legionella antigen was found positive and antibiotics were switched to levofloxacin. The patient required pressure support for ten days and was extubated on the fifteenth day of hospitalization.

\section{Epidemiology and clinical manifestations}

First identified in 1976 during an outbreak at the annual meeting of the American Legion in Philadelphia (where out of 221 people infected, 34 died), Legionella is a facultative intracellular aerobic gram negative rod producing beta-lactamase, hardly visible on Gram stain due to its diminutive size. There are two major presentations of Legionella infection; the first being Legionnaires' Disease which is a community acquired pneumonia whose course can become very dramatic and the second being Pontiac fever

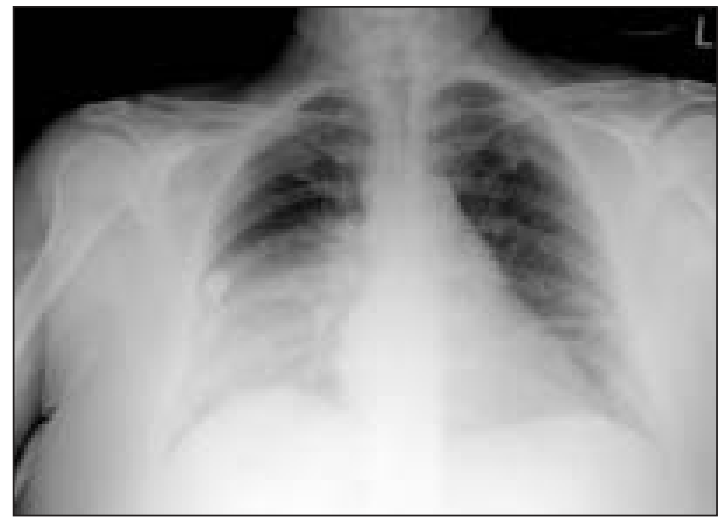

Figure 1. Chest X-ray revealing dense alveolar opacity at the right lung base with air bronchograms. There are left mid lung atelectatic changes and significant central vascular pulmonary congestion.

which is a more benign and self limited disease manifested through headaches, low grade fevers, malaise and chills without respiratory complaints and no radiological $x$-ray findings. Pneumonia is the most common clinical manifestation and Legionella has been reported as the third or fourth most commonly identified pathogen in community acquired pneumonias. ${ }^{1-2}$ However, it did account for only $4.7 \%$ of the CAP cases in one large study. ${ }^{3}$ Its prevalence is highlighted by the fact that it is also mentioned as one of the most common pathogens identified in nosocomial pneumonia.

\section{Legionnaires' Disease}

With an incubation period of two to ten days, this pathogen is considered transmitted through to the air conditioning and water cooling systems, rather then transmitted from person to person. Although first thought to manifest only as severe pneumonia (second only to S. pneumoniae in organisms identified in ICU admissions for pneumonia) accompanied by gastrointestinal symptoms and high fevers ${ }^{4-6}$, current diagnostic testing proved that presentation of Legionella infection may vary widely and that symptoms may be nonspecific.

Among the clinical clues for diagnosis of Legionnaires' disease are the gastrointestinal symptoms, especially diarrhea; neurological findings such as confusion, headache and lethargy; and fever > 390 C. Cough is usually mild and only slightly productive, hemoptysis is rarely encountered, and chest pain is infrequent. Physical exam is nonspecific with rales and subsequent signs of consolidation, fever as high as $40^{\circ} \mathrm{C}$, bradycardia, lethargy, and stupor. Sputum gram stain shows WBC abundance but scant or no microorganisms. Hyponatremia $(\mathrm{Na}+\text { often less than } 130)^{1,5-6,8}$, hepatic and renal dysfunction, hematuria, thrombocytopenia and failure to respond to classic $ß$ lactam antibiotic treatment are often specifically seen in patients with Legionella. One should always maintain a higher index of suspicion for Legionella infection in patients considered at risk including smokers, those with chronic 


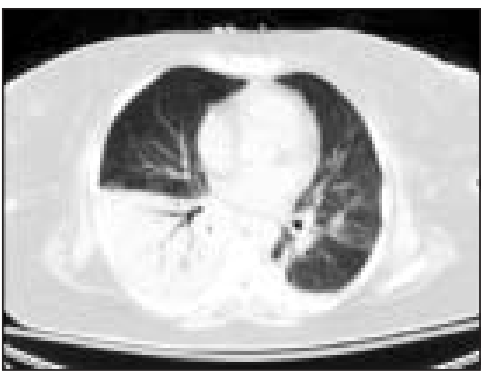

Figure 2. CT of the chest showing dense right lower lobe consolidation, scattered areas of groundglass opacity and scattered focal nodular densities.

lung disease, and immunosupressed patients such as those receiving steroids or anti-rejection drugs for post-organ transplant.

Radiological findings are usually already present by the third day of illness. Most commonly seen are patches consistent with unilobar infiltrates which may progress to consolidation. Pleural effusion may also be seen on $x$-ray, although infrequently'. In the immunosupressed host, densities may appear as round opacities at the lung base, often progressing into cavitary lesions. Of note, radiological changes usually lag behind the clinical course, with complete resolution of the infiltrates within 4 weeks to several months from the debut of the illness.

Although some clinical manifestions are distinctive for Legionella infection, none of them are pathognomonic or highly specific. Early detection with prompt and appropriate antibiotic treatment have been proven to save lives ${ }^{10,11}$. Culturing for Legionella spp. is the single most important laboratory test, but is time consuming and requires adequate respiratory specimen and a special culture media. Other tests have proved to be more beneficial: urinary antigen testing is rapid, sensitive, specific, and not costly. However, it is only useful for the diagnosis of L. pneumophila type 1 infection (accounting for 90 percent of communityacquired Legionella infections in the United States). The combination of culture of an appropriate respiratory specimen and urinary antigen testing are optimal as a diagnostic approach. Serologic tests, although available, are generally far less useful for the diagnosis of an individual patient but very useful for large epidemiological studies. While PCR-based tests exist, to date they do not exceed the sensitivity of culturing the organism. Given the severity and the high incidence of Legionella infection it is reasonable to perform specific diagnostic tests for all patients requiring hospitalization for community acquired pneumonia.

\section{Treatment}

The mortality of untreated or inadequately treated community acquired Legionnaires' disease is $16-30 \%$, while the mortality for the nosocomial Legionnaire's disease may be as high as $50 \%{ }^{12}$. Timely use of the current diagnostic tests as well as prompt antibiotic treatment with active drugs have decreased mortality to less than $10 \%$.

Although frequently used in the past, erythromycin has now been supplanted by newer macrolides as well as respiratory tract quinolones. Two studies ${ }^{13,14}$ showed the higher efficacy of levofloxacin compared to macrolides as well as fewer complications and shorter hospitalizations. Current recommendations are a 7-10 day course of azithromycin or 10-14 day course of levofloxacin, with a longer 21 day course for immunosupressed patients and patients requiring ICU admission. Given the high incidence and the severity of this disease, current recommendation for community acquired pneumonia requiring hospitalization is azithromycin either as monotherapy or along with $\beta$-lactame antibiotics ${ }^{15,16}$. Monotherapy with an active quinolone is also acceptable. For nosocomial pneumonia, a quinolone (ciprofloxacin or levofloxacin) is the empiric drug of choice since the nosocomial infections are frequently associated with gram negative bacilli. For endocarditis and other extrapulmonary infections, a combination therapy is recommended with levofloxacin or azithromycin plus rifampin. Despite a significant decrease in mortality with prompt and proper antibiotic treatment, patients often have residual symptoms 17 such as chronic fatigue syndrome (75\%) and residual neurological deficits (63\%).

Our case patient finished 21-day course treatment with levofloxacin for ARDS secondary to Legionnaires' disease. Hospital coursee was complicated by development of quadriparesis thought to be secondary to ICU neuropathy. Of note, Legionella Pneumophilla has also been linked independently with quadraparetic complications. The patient did achieve further improvement in her neurological status and was able to be transferred to a rehabilitation facility.

\section{References}

1. Fang, GD, Fine, M, Orloff, J, et al. New and emerging etiologies for community-acquired pneumonia with implications for therapy: a prospective multicenter study of 359 cases. Medicine 1990; 69:307.

2. Stout, JE, Yu, VL. Legionellosis. N Engl J Med 1997; 337:682.

3. Yu, VL, Greenberg, RN, Zadeikis, N, et al. Levofloxacin efficacy in the treatment of community-acquired legionellosis. Chest 2004; 125:2135.

4. Fraser, DW, Tsai, T, Ornstein, W, et al. Legionnaires' disease: description of an epidemic of pneumonia. N Engl J Med 1977; 297:1189.

5. Kirby, BD, Snyder, KM, Meyer, RD, Finegold, SM. Legionnaires' disease: report of sixty-five nosocomially acquired cases of review of the literature. Medicine (Baltimore) 1980; 59:188.

6. Mulazimoglu, L, Yu, VL. Can Legionnaires disease be diagnosed by clinical criteria? A critical review. Chest 2001; 120:1049.

7. Roig, J, Aguilar, X, Ruiz, J, et al. Comparative study of Legionella pneumophila and other nosocomial-acquired pneumonias. Chest 1991; 99:344.

8. Yu, VL, Kroboth, FJ, Shonnard, J, et al. Legionnaires' disease: new clinical perspective from a prospective pneumonia study. Am J Med 1982; 73:357.

9. Tan, MJ, Tan, JS, Hamor, RH, et al. The radiologic manifestations of Legionnaire's disease. The Ohio Community-Based Pneumonia Incidence Study Group. Chest 2000; 117:398.

10. Lepine, LA, Jernigan, DB, Butler, JC, et al. A recurrent outbreak of nosocomial legionnaires' disease detected by urinary antigen testing: evidence for long-term colonization of a hospital plumbing system. Infect Control Hosp Epidemiol 1998; 19:905.

11. Heath, CH, Grove, DI, Looke, DFM. Delay in appropriate therapy of Legionella pneumonia associated with increased mortality. Eur J Clin Microbiol Infect Dis 1996; 15:286.

12. Fraser, DW, Tsai, TR, Orenstein, W, et al. Legionnaires' disease: Description of an epidemic of pneumonia. N Engl J Med 1977; 297:1189.

13. Blazquez Garrido, RM, Espinosa Parra, FJ, Alemany Frances, L, et al. Antimicrobial chemotherapy for legionnaires disease: levofloxacin versus macrolides. Clin Infect Dis 2005; 40:800.

14. Mykietiuk, A, Carratala, J, Fernandez-Sabe, N, et al. Clinical outcomes for hospitalized patients with Legionella pneumonia in the antigenuria era: the influence of levofloxacin therapy. Clin Infect Dis 2005; 40:794.

15. Bartlett, JG, Dowell, SF, Mandell, LA, et al. Practice guidelines for the management of community-acquired pneumonia in adults. Clin Infect Dis 2000; 31:347.

16. Niederman, MS, Mandell, LA, Anzueto, A, et al. Guidelines for the Management of Adults with Community-acquired Pneumonia. Diagnosis, assessment of severity, antimicrobial therapy, and prevention. Am J Respir Crit Care Med 2001; 163:1730. 